\title{
Cerebrovascular Drug-Eluting Stent versus Bare-Metal Stent in the Treatment of Vertebral Artery Stenosis: A Non-Inferiority Randomized Clinical Trial
}

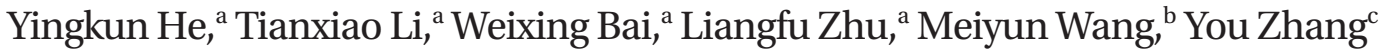 \\ ${ }^{a}$ Cerebrovascular Division, Interventional Department, Henan Provincial People's Hospital, Zhengzhou University, Zhengzhou, China \\ ${ }^{b}$ Radiology Department, Henan Provincial People's Hospital, Zhengzhou University, Zhengzhou, China \\ 'Epidemiology Center, Henan Provincial People's Hospital, Zhengzhou University, Zhengzhou, China
}

\section{Dear Sir:}

Drug-eluting coronary stents have been used for patients with vertebral artery stenosis to prevent the occurrence of in-stent restenosis which affects the therapeutic efficacy of angioplasty and stenting. However, the results are inconsistent. ' A new cerebrovascular sirolimus-eluting stent system (Maurora, Alain Biotechnology Co. Ltd., Beijing, China), ${ }_{1}^{2}$ different from the Apollo stent systems (Apollo, MicroPort Scientific Corp., Shanghai, China) made of 316L stainless steel, ${ }^{3}$ adopts $\mathrm{L} 605$ cobalt-chromium alloy that offers a higher yield and has tensile strength characteristics allowing for thinner thickness while maintaining adequate strength and flexibility suitable for a curved artery of the brain, and the sirolimus can reduce the cell proliferation. This trial was to evaluate the safety and efficacy of this new drug-eluting stent.

\section{Table 1. The inclusion and exclusion criteria}

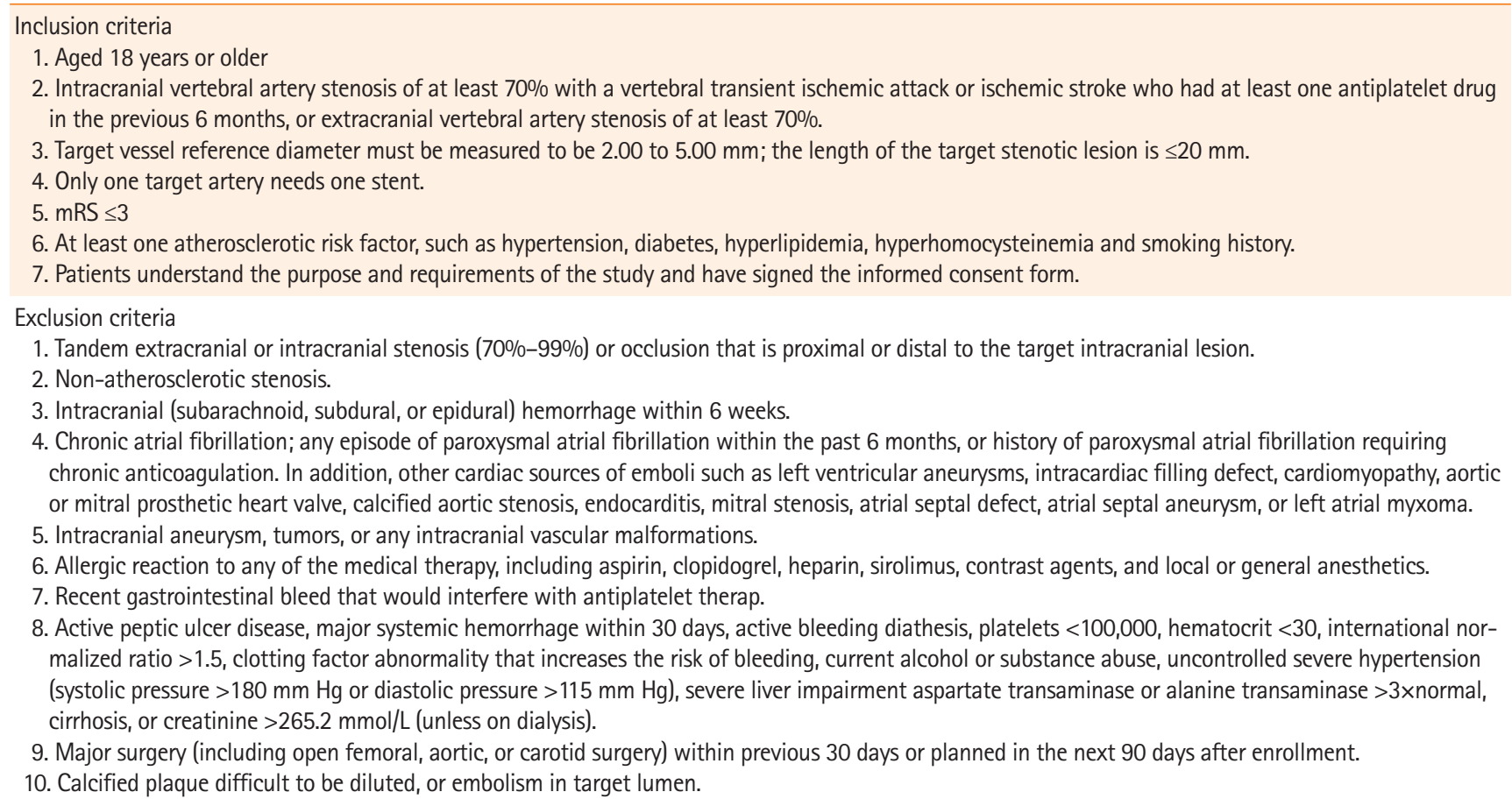


Table 2. Baseline and procedure-related characteristics

\begin{tabular}{|c|c|c|c|c|}
\hline Parameter & DES-G $(n=20)$ & BMS-G $(n=20)$ & Difference $(95 \% \mathrm{Cl})$ & $P$ \\
\hline Age (yr) & $58.6 \pm 10.6$ & $61.6 \pm 8.4$ & $-3.0(-9.1$ to 3.2$)$ & 0.335 \\
\hline Male sex & $16(80.0)$ & $16(80.0)$ & $0(-24.8$ to 24.8$)$ & 1.000 \\
\hline $\mathrm{BMI}$ & $24.4 \pm 2.7$ & $25.4 \pm 2.9$ & $-1.0(-2.9$ to 0.7$)$ & 0.240 \\
\hline Hypertension & $13(65.0)$ & $18(90.0)$ & $-25.0(-49.7$ to -0.3$)$ & 0.127 \\
\hline Hyperhomocysteinemia & $7(35.0)$ & $6(30.0)$ & $5.0(-24.0$ to 34.0$)$ & 1.000 \\
\hline Hyperlipidaemia & $3(15.0)$ & $3(15.0)$ & $0(-22.1$ to 22.1$)$ & 1.000 \\
\hline Diabetes mellitus & $4(20.0)$ & $7(35.0)$ & $-15.0(-42.3$ to 12.3$)$ & 0.480 \\
\hline Smoker & $9(45.0)$ & $9(45.0)$ & 0 (-30.8 to 30.8$)$ & 1.000 \\
\hline Coronary artery disease & $4(20.0)$ & $3(15.0)$ & $5.0(-18.5$ to 28.5$)$ & 1.000 \\
\hline Peripheral artery atherosclerosis & $7(35.0)$ & $10(50.0)$ & $-15.0 \%(-45.3$ to 15.3$)$ & 0.523 \\
\hline Recent qualifying event & & & NA & 0.005 \\
\hline Stroke & $5(25.0)$ & $11(55.0)$ & & \\
\hline TIA & $5(25.0)$ & $8(40.0)$ & & \\
\hline Others & $10(50.0)$ & $1(5.0)$ & & \\
\hline \multicolumn{5}{|l|}{$\begin{array}{l}\text { Location of the target stenosis in the } \\
\text { vertebral artery }\end{array}$} \\
\hline $\mathrm{V} 1-\mathrm{V} 3^{*}$ & $17(85.0)$ & $8(40.0)$ & 45.0 (18.4 to 71.6$)$ & 0.008 \\
\hline Time from qualifying event to procedure & $35(15-36)$ & $19(9-25)$ & NA & 0.488 \\
\hline $\mathrm{mRS}$ score & & & NA & 0.742 \\
\hline 1 & 16 & 16 & & \\
\hline 2 & 4 & 3 & & \\
\hline 3 & 0 & 1 & & \\
\hline Arterial target stenosis (\%) & $80(75-85)$ & $82.5(70-90)$ & NA & 0.535 \\
\hline Referenced normal artery diameter $(\mathrm{mm})$ & $3.8 \pm 0.8$ & $3.4 \pm 0.6$ & $0.4(-0.05$ to 0.86$)$ & 0.078 \\
\hline MORI & & & NA & 0.823 \\
\hline A & 11 & 9 & & \\
\hline B & 5 & 10 & & \\
\hline $\mathrm{C}$ & 4 & 1 & & \\
\hline Stent length & $12(12-14)$ & $13(10.5-13)$ & NA & 0.236 \\
\hline Balloon expansion time (sec) & $20(15-30)$ & $30(20-34)$ & NA & 0.158 \\
\hline Residual stenosis & $10(2.5-10)$ & $7.5(2.5-10)$ & NA & 0.822 \\
\hline
\end{tabular}

Values are presented as mean \pm SD, number (\%), or median (range).

DES-G, drug-eluting stent group; BMS-G, bare-metal stent group; Cl, confidence interval; BMI, body mass index; NA, not applicable; TIA, transient ischemic attack; mRS, modified Rankin Scale; MORI, Mori type.

*V1-V3, the extracranial segments of the vertebral artery.

This was a single center, open-label, prospective, non-inferiority, randomized, controlled trial (ChiCTR-IIR-16009115). ${ }^{2}$ Inclusion and exclusion criteria were based on the Endovascular Interventional Treatment for Ischemic Stroke Guideline of China (Table 1). Patients aged 18 years or older with intracranial vertebral artery stenosis of at least $70 \%$ and presence of transient ischemic attack or ischemic stroke were enrolled. And, for the patients with extracranial vertebral artery stenosis of at least 70\%, presence of symptoms was not necessary. The sample size of this non-inferiority trial was calculated based on our previous retrospective data ${ }^{4}$ and the non-inferiority $\operatorname{margin}^{2}(\Delta$ $=6 \%$ ). Patients were randomized in a 1:1 ratio to undergo stenting with Maurora stents or Apollo stents between September 2014 and September 2015.

Primary outcomes included surgical complications within 30 days after procedure and the incidence of in-stent restenosis within 6 months after operation. Secondary outcomes included stroke ipsilateral to the target vertebral artery cerebrovascular and cardiovascular events, and serious adverse events within 12 months after operation. In-stent restenosis was defined as 
Table 3. Primary and secondary outcomes 30-day and 1-year after procedure

\begin{tabular}{|c|c|c|}
\hline Outcome & DES-G $(n=20)$ & BMS-G $(n=20)$ \\
\hline \multicolumn{3}{|l|}{ Primary outcomes (\%) (95\% Cl) } \\
\hline Procedure complications within 30 days & 0/20 (0) (0-13.9) & 0/20 (0) (0-13.9) \\
\hline In-stent restenosis & $1 / 20(5)(0.1-24.9)$ & $5 / 20(25)(8.7-49.1)$ \\
\hline \multicolumn{3}{|l|}{ No. of secondary outcomes } \\
\hline All adverse events within 30 days & 0 & 0 \\
\hline All adverse events beyond 30 days & 2 & 12 \\
\hline Death & 0 & 0 \\
\hline Stroke & 0 & 2 \\
\hline In-stent restenosis & 1 & 5 \\
\hline Symptomatic & 0 & 2 \\
\hline Angina & 1 & 1 \\
\hline Dizziness & 0 & 2 \\
\hline Lower limbs paresthesia & 0 & 1 \\
\hline Gum bleeding & 0 & 1 \\
\hline \multicolumn{3}{|c|}{ Post hoc outcomes after complete follow-up (\%) (95\% Cl) } \\
\hline Stroke in target vertebral artery territory & 0/20 (0) (0-13.9) & $2 / 20(10)(1.2-31.7)$ \\
\hline
\end{tabular}

DES-G, drug-eluting stent group; BMS-G, bare-metal stent group; $\mathrm{Cl}$, confidence interval.

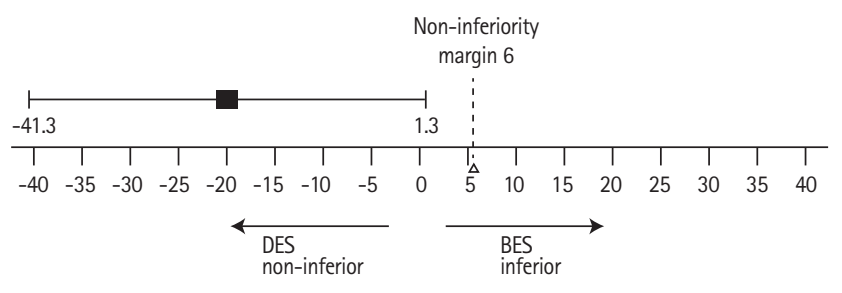

Figure 1. The non-inferiority text for restenosis. DES, drug-eluting stents; BMS, bare-metal stents.

a lesion demonstrating more than 50\% stenosis (within or immediately [within $5 \mathrm{~mm}$ ] adjacent to the stent) and more than $30 \%$ absolute luminal loss at 6-month angiographic follow-up imaging (30\% increase in posttreatment stenosis).

As a result, 40 enrolled patients were randomly divided into the two groups to receive stenting with either Maurora stent (drug-eluting stent group [DES-G], $n=20$ ) or Apollo stent (baremetal stent group [BMS-G], $n=20$ ), with no cross-over. Though the study groups were well balanced with regards to the baseline and procedure-related demographics data, which showed no significant differences ( $P>0.05)$, recent qualifying event $(P=0.005)$ and location of target stenosis in the vertebral artery $(P=0.008)$ showed statistical significance (Table 2). All the primary and secondary outcomes can be seen in Table 3. No procedure-related complications occurred within 30 days after procedure. The median angiography follow-up time was 6.5 months in DES-G and 6.4 months in BMS-G. In-stent resteno- sis rates were $5 \%$ in the DES-G and 25\% in BMS-G with a difference of $-20 \%(P=0.182)$, demonstrating non-inferiority (Figure 1).

The mean clinical follow-up was 18.0 months for DES-G and 18.8 months for BMS-G. Serious adverse events occurred in one patient in the DES-G and three in the BMS-G $(P=0.605)$. The 1 -year incidence rates for cerebrovascular and cardiovascular events were $5 \%$ in DES-G and 15\% in BMS-G (log-rank test, $P=0.317$ ). The 1 -year incidence rates for ipsilateral stroke were $0 \%$ in DES-G and $10 \%$ in BMS-G (log-rank test, $P=0.152$ ).

In this study, two strokes occurred all due to in-stent restenosis, which demonstrated that in-stent restenosis was one important factor that affected the stenting efficacy. Non-inferiority test used in the study reduced the the need of large sample size, but the basic characteristics between the two groups were not well balanced, and it failed to obtain a superior result in the decrease in the in-stent stenosis. However, as far as we know, this study was the first randomized controlled trial that used special cerebrovascular drug-eluting stent for treating vertebral artery stenosis. The results showed that the cerebrovascular drug-eluting stent for the treatment of vertebral artery stenosis was safe, and was not inferior to the bare metal stent in reducing the restenosis rate. Although statistically insignificant, it showed a tendency to reduce the incidence of restenosis (5\% and 25\%). This study has laid the foundation for phase III multicenter clinical trial in the future. 


\section{References}

1. Wabnitz A, Chimowitz M. Angioplasty, stenting and other potential treatments of atherosclerotic stenosis of the intracranial arteries: past, present and future. J Stroke 2017;19: 271-276.

2. He Y, Li T, Bai W, Zhu L, Wang Z, Xue J, et al. [The protocol of cerebrovascular drug-eluting stents verse bare metal stents in the treatment of vertebral artery stenosis]. Chin J Int Radiol (Electr Ed) 2016;2:1-6. Chinese.

3. Jiang WJ, Xu XT, Jin M, Du B, Dong KH, Dai JP. Apollo stent for symptomatic atherosclerotic intracranial stenosis: study results. AJNR Am J Neuroradiol 2007;28:830-834.

4. Zhao,T, Li Z, Xue J, Li T, Feng G, Zhang L, et al. [Control study of drug-eluting stent versus bare metal stent in treating vertebral artery ostial stenosis]. Chin J Neuromedicine 2015;14:506510. Chinese.

Correspondence: Tianxiao Li

Cerebrovascular Division, Interventional Center Henan Provincial People's Hospital, Zhengzhou University, No. 7, Weiwu Road, Zhengzhou 450000, China Tel: +86-371-65580265

Fax: +86-371-65964376

E-mail:dr.litianxiao@henu.edu.cn

Received: February 12, 2018

Revised: March 15, 2018

Accepted: April 6, 2018

This study has received funding by The National Natural Science Foundation of China (No. 81601583), The Technology Research Project of Henan Province (No. 162102310268 and 172102310509) and Aboard Research Project of Henan Provincial Health Commission (2016054). 